\title{
TEMPERATURE DEPENDENCE OF THE ROTATIONAL MAGNETIC ANISOTROPY IN AMORPHOUS Gd-Co FILMS
}

\author{
G. V. KURLYANDSKAYA* and A. V. SVALOV \\ Institute of Physics and Applied Mathematics, Ural State University, Ekaterinburg 620083, Russia \\ *Institute of Metal Physics, Russian Academy of Sciences (Ural Branch), Ekaterinburg, Russia
}

\begin{abstract}
The phenomenon of rotational magnetic anisotropy in the plane of amorphous Gd-Co films was observed. The saturation magnetization, perpendicular and rotational anisotropy constants, torque curves were investigated for different composition films. The experimental and theoretical results suggest that a fine stripe domain structure existed in transcritical films is responsible for rotational magnetic anisotropy.

KEYWORDS: ANISOTROPY, AMORPHOUS FILMS, RARE EARTH-TRANSITION METAL ALLOYS, MAGNETIC TORQUE, DOMAIN STRUCTURE
\end{abstract}

The phenomenon of rotational magnetic anisotropy (RMA) in the plane of the film has well been studied on polycrystalline films of transition metals and their alloys $[1,2]$. A sufficiently large magnetic field applied in the plane of the film creates an easy axis of the rotational anisotropy. Observations of RMA in other materials have also been reported lately: dilutely dispersed $\mathrm{Co}-\mathrm{C}-\mathrm{O}$ films [3], amorphous $\mathrm{Tb}-\mathrm{Fe}$ [4].

In the present investigation RMA has been studied in amorphous ferrimagnetic Gd-Co films. It has been experimentally shown that the emergence of RMA is connected with the transition of the $f j .1 \mathrm{~m}$ into a "transcritical" state.

Amorphous $\mathrm{Gd}-\mathrm{Co}$ films $1 \mu \mathrm{m}$ thick, having different compositions, were prepared by r.f. sputtering on water-cooled glass substrates. The saturation magnetisation $M_{S}$, perpendicular and rotational anisotropy constants $K_{u}$ and $K_{R}$ were measured by a torque method.

Figure 1 shows the torque curves measured for a sample with compensation temperature $T_{\mathrm{K}}<77 \mathrm{~K}$ at room temperature for various values of the magnetic field rotated in the film plane. The film was magnetized preliminarily in its own plane by a field of $80 \mathrm{kA} / \mathrm{m}$. The direction of the rotary field was denoted by $\theta$ and was measured with respect to the magnetisation field direction.
At present two points of view on the nature of the RMA are known best. One of them supposes that high-anisotropy centers exist in the films [5]. According to the other it is supposed that a fine stripe domain structure exists in trans-

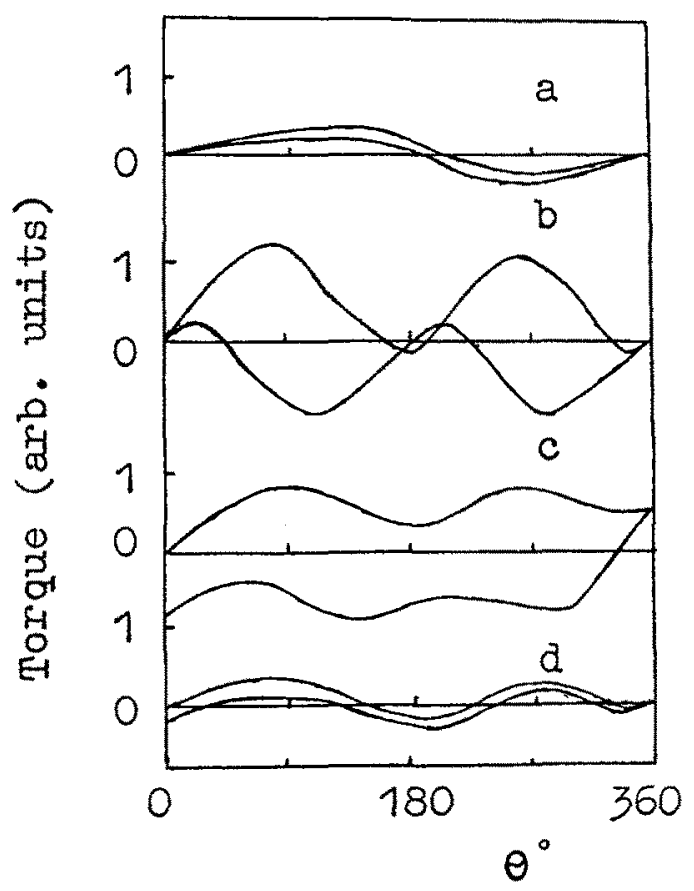

Fig.1. Torque as a function of $\theta$ for certain applied fields $(\mathrm{a}-6 \mathrm{kA} / \mathrm{m} ; \mathrm{b}-16 \mathrm{kA} / \mathrm{m} ; \mathrm{c}-40 \mathrm{kA} / \mathrm{m}$; $\mathrm{d}-80 \mathrm{kA} / \mathrm{m}$ ) at room temperature for the sample with $T_{k}<77 \mathrm{~K}$. 
critical films. This structure is responsible for the RMA $[2,6]$. The film state is called "transcritical", if $Q=K_{u} / 2 \pi M_{S}^{2}<1$ and film thickness

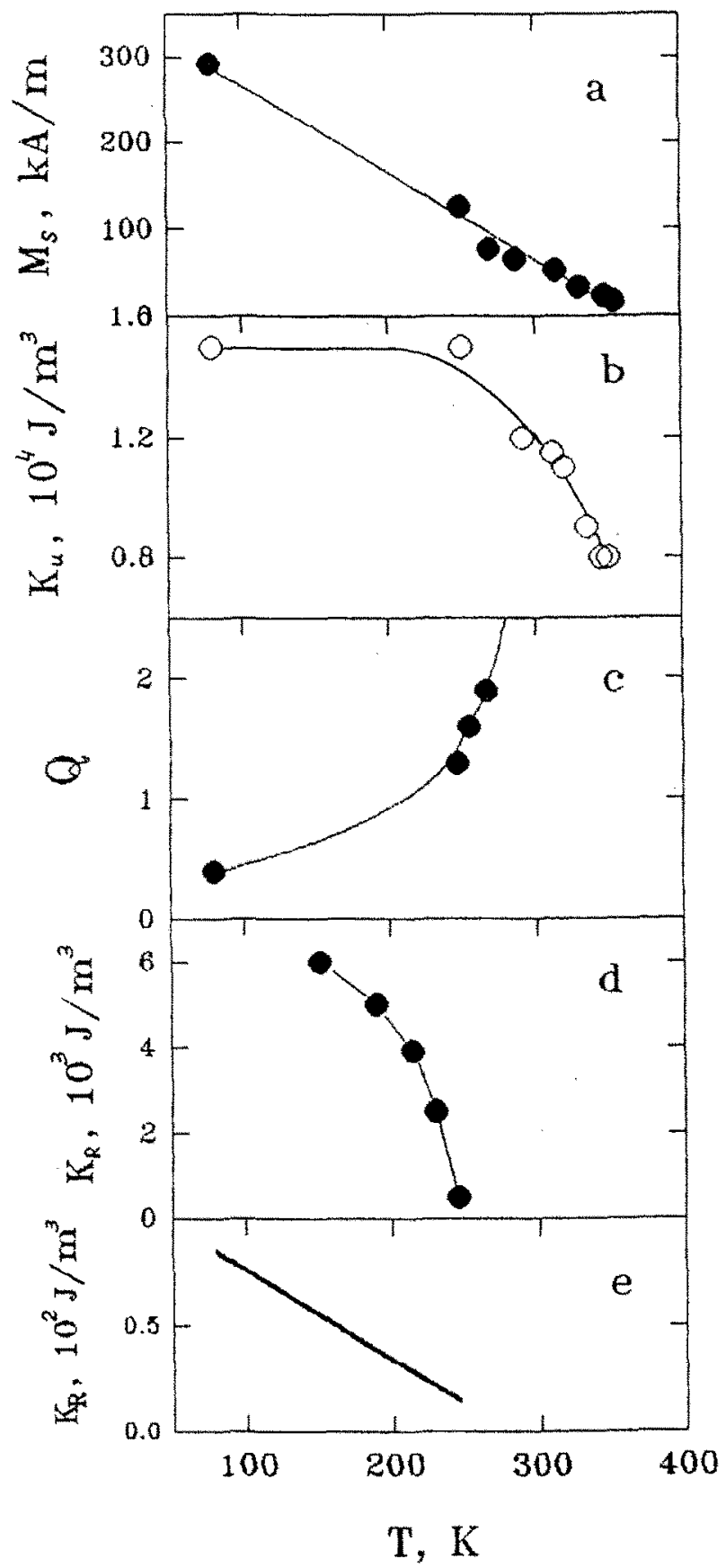

Fig.2. Temperature dependence of the saturation magnetizatiom $M_{S}(a)$, perpendicular anisotropy constant $K_{u}(b)$, quality factor $Q(c)$, rotatable anisotropy constant $K_{R}$ : experimental data (d), calculated data (e). $\ell>\ell_{c}=2 \pi \sqrt{\mathrm{A} / \mathrm{K}_{u}}$, where $\mathrm{A}$ is the exchange interaction parameter.

RMA in the film plane was observed in our experiment in the sample with compensation temperature $T_{K}=370 \mathrm{~K}$ at a temperature lower than $250 \mathrm{~K}$ (Fig.2d), since this sample passed into transcritical state at the temperature of $210 \mathrm{~K}$ (Fig.2c).

Noncoincidence of the temperature of transition of film into the transcritical state and that of emergence of RMA may be connected with the sample chemical inhomogeneity. There is a transition region, similar to a $90^{\circ}$ domain wall, between the oxidized surface layer with easy magnetisation plane anisotropy and the main layer with perpendicular anisotropy. The transition of the film into the transcritical state possibly takes place not simultaneously over the entire volume of the film, but gradually by widening of the subsurface domain wall within a certain temperature interval [7] The temperature dependence of $K_{R}$, estimated in accordance with the model [2], is in a qualitative agreement with the experimental one, but the observed values of $K_{R}$ are about ten times as great as the calculated values (Fig.2e). Perhaps, the model developed for polycristalline films is not fully applicable to the amorphous films.

[1] R.J.Prosen,J.0.Holmen and B.E.Gran: J.Appl.Phys., 32, 915 (1961).

[2] A.A.Glaser, I.Konstantinova, A.P.Potapov and R.I.Tagirov: Phys.Met.Metal1. 33(5), 43 (1972).

[3] R.D.Kangiev, I.K. Igumenov, A.N.Podmarkov and G.V.Popov:Solid State Comm., $72(11), 1101$ (1989).

[4] M.J.O' Shea and A.Fert:Europhys. Lett. 9(3), 283 (1989).

[5] M.S.Cohen:J.App1.Phys., 33(10), 2968 (1962).

[6] H.Fujiwara, Y.Sugita and N.Saito: Appl.Phys.Letters, 4(12), 199 (1964).

[7] V.O.Vas'kovskiy, G.S.Kandaurova, Ye.G.Gerasimov, V.Kh.Osadchenko, A.Svalov and Ye.M.Pampura:Phys.Met. Metal1, 71(2), 82 (1991). 